ERR AT U M

Corrigendum to Volume 12, Number 4, pp. 278-286

Published online: 29 April 2010

CFESEO 2010

\title{
Current controversies in the management of breast cancer
}

\author{
Ignasi Tusquets · Laura García-Estévez • Encarna Adrover • Lourdes Calvo • Isabel Álvarez • \\ Jesús García Mata · Yolanda Fernández • Mireia Margueli • Miquel Ángel Seguí · César Rodríguez • \\ Álvaro Rodríguez Lescure · Ramon Colomer · Pere Gascón · Miguel Martín · Emilio Alba • \\ Agusti Barnadas • Antonio Llombart • Joan Albanell • Ana Lluch
}

There was a mistake in the list of authors of the article entitled "CURRENT CONTROVERSIES IN THE MANAGEMENT OF BREAST CANCER". The name of author Montserrat Muñoz was omitted by mistake. The correct list of authors is:

I. Tusquets ${ }^{1}$, L. García-Estévez², E. Adrover ${ }^{3}$, L. Calvo ${ }^{4}$, I. Álvarez ${ }^{5}$ J. García Mata ${ }^{6}$, Y. Fernández $^{7}$, M. Margueli ${ }^{8}$, M.A. Seguî ${ }^{1}$, M. Muñoz ${ }^{10}$, C. Rodríguez ${ }^{11}$, A. Rodríguez Lescure ${ }^{12}$, R. Colomer $^{13}$, P. Gascón ${ }^{10}$, M. Martín ${ }^{14}$, E. Alba ${ }^{15}$, A. Barnadas $^{8}$, A. Llombart ${ }^{16}$, J. Albanell ${ }^{1}$, A. Lluch ${ }^{17}$

\section{AFFILIATIONS:}

${ }^{1}$ Departments of Medical Oncology, Hospital del Mar-IMAS, Barcelona, Spain

${ }^{2}$ Centro Integral Oncológico Clara Campal, Madrid, Spain

${ }^{3}$ Hospital de Alicante, Alicante, Spain

${ }^{4}$ Complejo Hospitalario Universitario Juan Canalejo, La Coruña, Spain

${ }^{5}$ Hospital Donostia, San Sebastián, Spain

${ }^{6}$ Complejo Hospitalario Ourense, Ourense, Spain

${ }^{7} \mathrm{HUCA}$, Oviedo, Asturias, Spain

${ }^{8}$ Institut Català d'Oncologia, Hospital Germans Trias i Pujol, Universitat Autónoma de Barcelona, Badalona, Barcelona, Spain

${ }^{9}$ Consorci Sanitari Parc Taulí, Sabadell, Barcelona, Spain

${ }^{10}$ Hospital Clínic Barcelona, Spain

${ }^{11}$ Hospital Universitario de Salamanca, Spain

${ }^{12}$ Hospital General Universitario de Elche, Spain

${ }^{13}$ Centro Oncológico MD Anderson España, Madrid, Spain

${ }^{14}$ Hospital Universitario San Carlos, Madrid, Spain

${ }^{15}$ Hospital Clínico Universitario, Málaga, Spain

${ }^{16}$ Hospital Universitario Arnau Vilanova, Spain

${ }^{17}$ Hospital Clínico Universitario, Valencia, Spain

The original version of the article can be found in:

Clin Trans Oncol (2010) 12:278-286

DOI 10.1007/s12094-010-0504-x 